\title{
RESISTENCIA A FUERZA CORTANTE DE COLUMNAS DE CONCRETO REFORZADAS CON CAMISAS DE ACERO
}

\author{
Oscar M. González Cuevas ${ }^{(1)}$, José Juan Guerrero Correa ${ }^{(1)}$, Bernardo Gómez González ${ }^{(1)}$, \\ Félix Alberto Flores Díaz ${ }^{(1)}$
}

\begin{abstract}
RESUMEN
Muchos edificios de concreto dañados por los sismos ocurridos en 1985 en la ciudad de México han sido reforzados colocando camisas de acero en sus columnas, que consisten de ángulos a lo largo de las aristas y placas o soleras transversales, sin que se tenga evidencia experimental del comportamiento de las columnas así reforzadas. Con base en lo anterior, se han realizado tres series de ensayes en columnas de concreto reforzadas con camisas de acero sujetas a carga axial y carga transversal alternante. El objetivo fue determinar si su comportamiento era adecuado y si su resistencia a fuerza cortante se podía calcular con los procedimientos usuales. Las variables estudiadas fueron el tamaño de los ángulos y las placas transversales que constituían la camisa, el daño previo a las columnas y distintos procedimientos constructivos: uso de resina epóxica para pegar los ángulos al concreto, sujetarlos a presión antes de soldar las soleras, soldar las soleras sin pegar ni presionar los ángulos, calentar las soleras y soldarlas antes de que se enfríen y combinaciones de estos procedimientos. Se describe el trabajo experimental y se presentan estudios analíticos para la interpretación de los ensayes.
\end{abstract}

\begin{abstract}
Many reinforced concrete buildings damaged by the Mexico City 1985 earthquakes were reinforced jacketing their columns with longitudinal steel angles in the corners and transverse steel plates. Since there is no evidence of the behavior of columns reinforced in this way, an experimental program was carried out in three series of specimens with constant axial load and cyclic reversal transverse load. The main objectives of the program were to determine if the behavior of the columns is adequate and if their shear resistance can be calculated using the common procedures. The variables of the tests were the size of the longitudinal angles and of the transverse plates, the previous damage of the columns and different jacketing methods: sticking the angles with epoxy resin, pressing the angles against the concrete before welding the plates, welding the plates neither sticking nor pressing the angles, welding the plates after heating them, and combinations of these procedures. The experimental work is described and an analytical interpretation of the results is included.
\end{abstract}

\section{INTRODUCCIÓN Y OBJETIVO}

A partir de 1985, muchos edificios dañados por los sismos ocurridos en ese año, o edificios que sin haber sufrido daños debieron ser reforzados, se han reparado y/o reforzado con camisas de acero,

\footnotetext{
Artículo recibido el 28 de febrero de 2006 y aprobado para su publicación el 13 de julio de 2007. Se aceptarán comentarios y/o discusiones hasta cinco meses después de su publicación.

(1) Universidad Autónoma Metropolitana-Azcapotzalco, Av. San Pablo 180, C.P. 02200, México, D.F., omgc@correo.azc.uam.mx, jigc@correo.azc.uam.mx
} 
consistentes en ángulos de acero colocados en las aristas verticales de las columnas, unidos mediante soleras transversales colocadas en toda la altura del elemento. No se tiene evidencia experimental de que el comportamiento de estas columnas corresponda a lo que se supone teóricamente y especialmente se tienen dudas de que los procedimientos constructivos usuales permitan lograr un trabajo satisfactorio del elemento original y su camisa (Iglesias et al. 1988). El Reglamento de Construcciones para el Distrito Federal y sus Normas Técnicas Complementarias (Gobierno del Distrito Federal, 2004)) no establecen una normatividad específica respecto a este tema, por lo que no se tienen disposiciones mínimas para llevar a cabo este tipo de rehabilitación.

Los procedimientos usados comúnmente en la práctica consisten en alguna de las siguientes modalidades: a) pegar las camisas de acero a las columnas de concreto con resina epóxica; b) apretar los ángulos longitudinales contra las columnas y soldarles las soleras transversales mientras se sostiene el apriete; c) colocar los ángulos longitudinales a lo largo de las aristas, calentar las soleras y soldarlas mientras están calientes, para que al enfriarse se contraigan y aprieten los ángulos contra las columnas; d) alguna combinación de los tres métodos anteriores.

En este artículo se presentan los resultados de un conjunto de ensayes encaminados a estudiar el comportamiento y la resistencia a fuerza cortante de columnas reforzadas de la manera antes descrita. El objetivo de la investigación fue obtener recomendaciones sobre los procesos constructivos más adecuados y sobre el cálculo de la resistencia a fuerza cortante de columnas encamisadas de la manera descrita. Otro objetivo importante fue verificar si columnas que habían sufrido daño previo, producido por fuerzas cortantes, podían recuperar o incrementar su resistencia a esta acción con la colocación de camisas de acero. Los especímenes de ensaye se sometieron a flexocompresión, con cargas cíclicas y con inversión en el signo de la fuerza que producía flexión y fuerza cortante, con objeto de simular la acción de sismos.

\section{DESCRIPCION DEL PROYECTO}

\section{Especímenes de ensaye}

Los especímenes de ensaye consistieron en columnas con una sección transversal de 20 X 25 cm y altura de $150 \mathrm{~cm}$, figura 1. El refuerzo longitudinal consistió en 4 varillas del No. 6 y los estribos fueron fabricados con varillas del No. 3. Ya que se buscaba que la fuerza cortante fuese resistida por el concreto y las soleras, los estribos se colocaron con una separación de $75 \mathrm{~cm}$. El concreto utilizado tenía una resistencia a la compresión nominal, $f_{c}^{\prime}$, de $200 \mathrm{~kg} / \mathrm{cm}^{2}$ y el acero un límite de fluencia nominal, $f_{y}$, de $4200 \mathrm{~kg} / \mathrm{cm}^{2}=$

El encamisado de acero consistió en ángulos colocados en las aristas de la columna unidos mediante soleras en toda la altura. El acero de ángulos y soleras tenía un límite de fluencia, $f_{y}$, de $2530 \mathrm{~kg} / \mathrm{cm}^{2}$. Los ángulos empleados fueron de dos tipos: ángulos cuya área transversal equivalía a la cuantía mínima de refuerzo longitudinal de la columna, y ángulos cuya área transversal, adicionada al refuerzo longitudinal, equivalían a la cuantía máxima de acero especificada en las Normas Técnicas Complementarias para Diseño y Construcción de Estructuras de Concreto (NTCC-04) (Gobierno del Distrito Federal, 2004). El ángulo utilizado para satisfacer la condición de cuantía mínima especificada fue uno de lados iguales de $3.2 \mathrm{~cm}$ de ancho y $0.3175 \mathrm{~cm}$ de espesor, con área transversal de $1.93 \mathrm{~cm}^{2}$ (LI 32X3, según notación del Instituto Mexicano de la Construcción en Acero, IMCA), y el ángulo especificado para la cuantía máxima fue uno de lados iguales de $6.4 \mathrm{~cm}$ de ancho y $0.635 \mathrm{~cm}$ de espesor, con área transversal de $7.68 \mathrm{~cm}^{2}$ (LI 64X6). Las soleras usadas con los ángulos pequeños fueron de $2.54 \mathrm{~cm}$ de ancho por $0.3175 \mathrm{~cm}$ de espesor; y las usadas con los ángulos grandes fueron de $2.54 \mathrm{~cm}$ ancho por $0.635 \mathrm{~cm}$ de espesor. La 
soldadura que unía las soleras a los ángulos se diseñó de tal manera que se desarrollara el esfuerzo de fluencia en aquellas sin que fallase la soldadura.

Las columnas se empotraron en sus extremos inferiores una longitud de $50 \mathrm{~cm}$. Las cargas vertical y horizontal se aplicaron en el extremo superior, de tal manera que la longitud de ensaye de $100 \mathrm{~cm}$ representaba la mitad de una columna de $200 \mathrm{~cm}$ a escala normal, cuyo punto de inflexión se supone a la mitad de su altura. Más adelante se describen las variables estudiadas y las características de los especímenes de acuerdo a las combinaciones de variables.

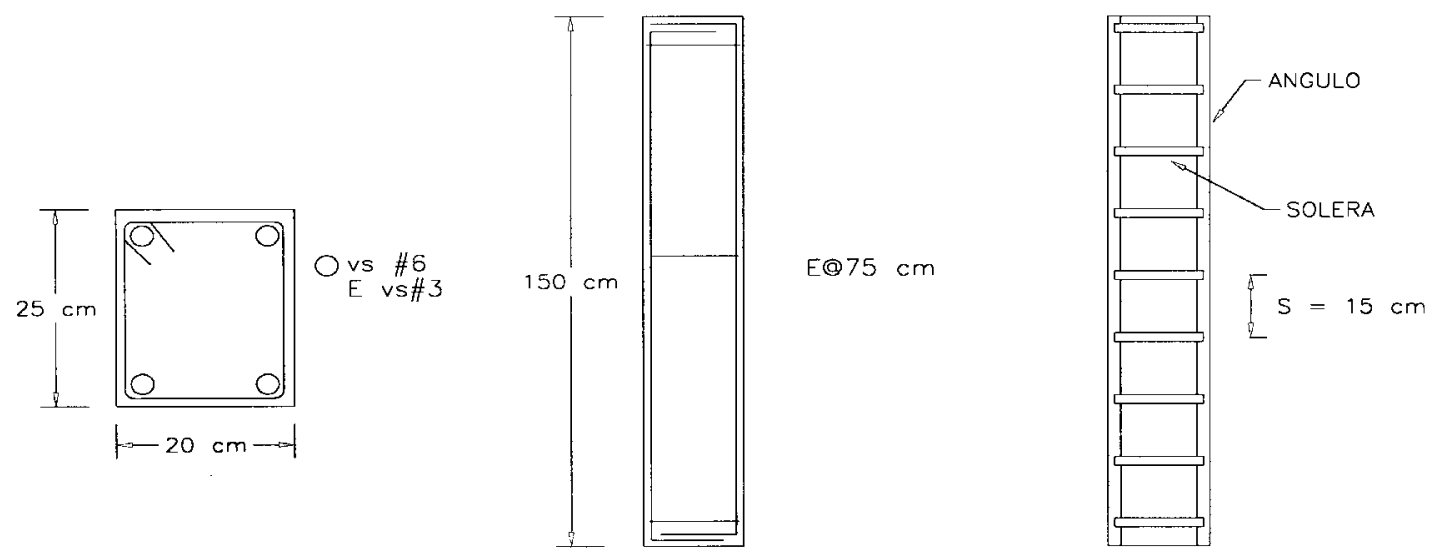
a) Elemento original
b) Elemento encamisado.

Figura 1. Elemento tipo

\section{Dispositivo de cargas}

Para llevar a cabo estas pruebas se utilizó un marco rígido de acero que permitía aplicar carga vertical y carga horizontal, esta última en dos sentidos, figura 2. Se diseñó un dispositivo para la parte superior de la columna que hacía posible mantener la verticalidad de la carga aunque la columna tuviese desplazamientos horizontales importantes (González Cuevas, O. et al. 1999). Este dispositivo es el señalado con el nombre de rótula cabezal (2) en la figura 2.

La carga lateral se transmitía a las columnas a través de un anillo de acero (5) colocado en la parte superior. De esta manera se podía empujar en un sentido y jalar en el otro sin introducir esfuerzos de tensión en el extremo del espécimen de ensaye. Para dar el empotramiento a la columna en su parte inferior, se fabricaron dos vigas de acero (8) que se colocaron sobre la viga inferior del marco de acero. Una de estas vigas se colocó en contacto con la columna por medio de una placa de acero, mientras que entre la otra viga y la columna se colocaron dos gatos hidráulicos que permitían mantener el empotramiento durante los ensayes. 
Previamente al montaje de la columna en el dispositivo de carga, se vertía azufre en la cara superior de la columna que recibiría la carga vertical, con el propósito de tener una superficie perfectamente horizontal y una distribución uniforme de la carga transmitida por el gato. En la figura 3 se presenta una vista general del ensaye de un espécimen típico.

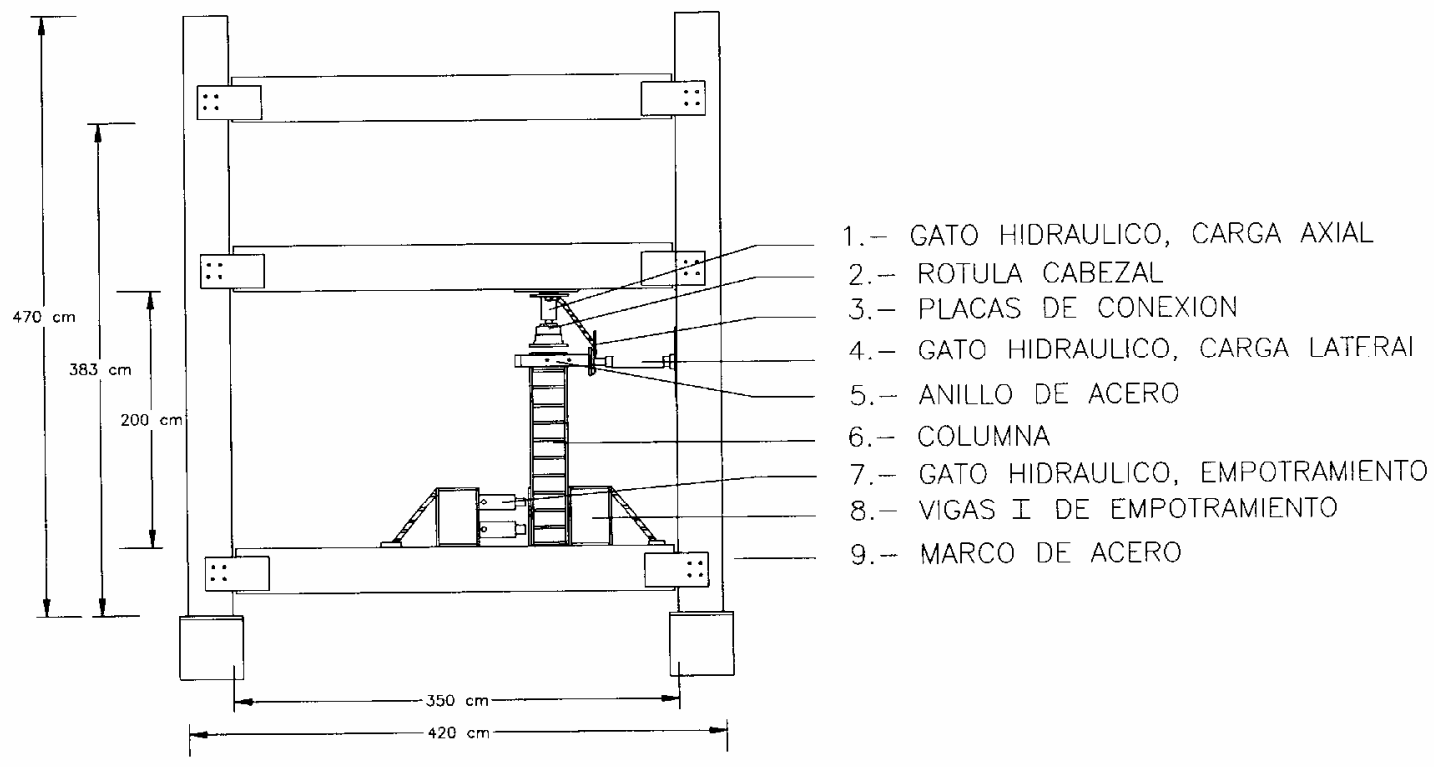

Figura 2. Marco de prueba, esquema general de ensaye

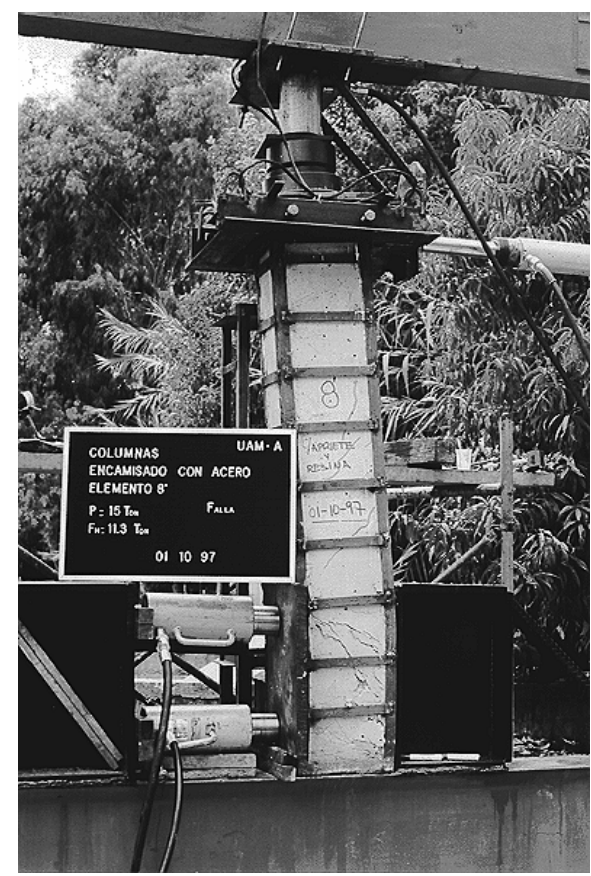

Figura 3. Vista general de un ensaye 


\section{Aplicación de cargas}

Las columnas se sometieron a cargas axial y lateral, por lo que el elemento quedó sujeto a flexocompresión y fuerza cortante. Primero se aplicó una carga axial de 15 ton, que corresponde al 15 por ciento de la resistencia a carga axial pura. Esta carga se mantuvo constante durante el ensaye, y posteriormente se aplicó la carga lateral en forma cíclica. Los seis primeros ciclos de carga se aplicaron de tal manera que la columna se vio sujeta a una fracción de su resistencia calculada, y los siguientes ciclos, a cargas que producían deformaciones laterales en el extremo de la columna correspondientes a cierto número de veces la deformación de fluencia. La carga lateral se fue incrementando de esta manera hasta obtener la falla del elemento.

Cada ciclo de carga consistió en aplicar carga lateral en un sentido, mantener esa carga durante dos minutos y al término, tomar la lectura de las deformaciones. Posteriormente la carga lateral se reducía a un valor nulo y al cabo del mismo periodo de tiempo se tomaba la lectura de las deformaciones. Después se aplicaba la carga lateral en sentido contrario y se repetía el procedimiento de toma de lecturas y descarga. De esta manera se proseguía el ensaye hasta la falla del espécimen. Además de las cargas y las deformaciones, se registraba el patrón de agrietamientos. Las fallas de todos los especímenes fueron del tipo de tensión diagonal, con agrietamiento inclinado que se desarrollaba en forma progresiva, es decir, los especímenes tuvieron cierta ductilidad por lo que se sobrepasó la deformación correspondiente a la fluencia del acero en tensión. Se pudo determinar que una grieta de $3 \mathrm{~mm}$ de ancho correspondía a un daño grave del espécimen y a la que se conoce como carga de agrietamiento inclinado (González Cuevas, 2005). Los especímenes que se dañaron previamente a su encamisado, se cargaron hasta que se formaron grietas de este ancho.

\section{Variables estudiadas}

La investigación se dividió en tres series de ensayes. En la primera, se incluyeron como variables el tamaño de los ángulos y las soleras que constituían las camisas y cuatro diferentes procesos de colocación de los ángulos sobre las columnas: pegarlos con resina epóxica, presionarlos sobre las columnas y soldarles las soleras, combinar los dos procedimientos anteriores, y colocarlos sin presión y sin resina y después soldar las soleras. De esta manera se tuvieron las siguientes ocho combinaciones de las variables estudiadas.

a) elemento con cuantía mínima, con presión y con resina

b) elemento con cuantía mínima, con presión y sin resina

c) elemento con cuantía mínima, sin presión y con resina

d) elemento con cuantía mínima, sin presión y sin resina

e) elemento con cuantía máxima, con presión y con resina

f) elemento con cuantía máxima, con presión y sin resina

g) elemento con cuantía máxima, sin presión y con resina

h) elemento con cuantía máxima, sin presión y sin resina

Se elaboraron 2 especímenes con cada una de estas combinaciones. Además, se elaboraron 3 elementos testigo sin encamisar, por lo que en esta primera serie se ensayaron 19 especímenes. A continuación se describe el procedimiento constructivo para las distintas combinaciones de variables. 


\section{Ángulos con presión y con resina}

Se colocaba la resina (Sikadur-31) a lo largo de las caras interiores de los ángulos con una espátula y después se fijaba el ángulo a la columna por medio de un anillo de acero que proporcionaba la presión necesaria para eliminar el sobrante de resina, figura 4.

Una vez que la resina había endurecido, se incrementaba la presión de los ángulos sobre las columnas apretando los tornillos del anillo de acero con un torquímetro hasta alcanzar una presión especificada. La fuerza que ejercía el anillo de acero sobre el ángulo era tal que dividida entre un área igual al cuadrado del ancho del ángulo no sobrepasaba al 75 por ciento del esfuerzo resistente al aplastamiento del concreto.

\section{Ángulos con presión y sin resina}

El proceso fue similar al anterior, únicamente que no se llevó a cabo el proceso de colocar resina epóxica en el ángulo para fijarlo al elemento de concreto.

\section{Ángulos sin presión y con resina}

Para este caso se aplicó la resina al ángulo y posteriormente se fijó al elemento de concreto aplicando únicamente la presión necesaria para eliminar el exceso de resina. Posteriormente se soldaron las soleras a los ángulos.

\section{Ángulos sin presión y sin resina}

Los ángulos se colocaron sobre las aristas de la columna de concreto y se sujetaron con el anillo de acero únicamente para mantenerlos en la posición adecuada mientras se soldaban las soleras.

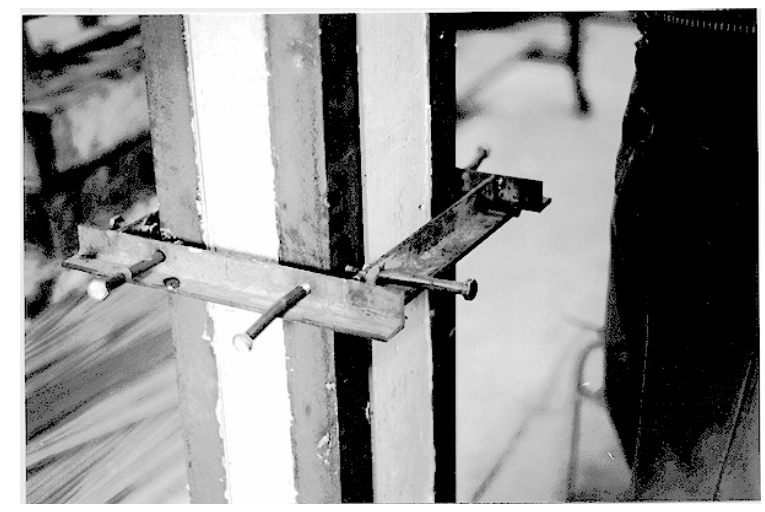

Figura 4. Colocación de los ángulos con un anillo de acero

En la segunda serie de ensayes, con base en los resultados de la primera, sólo se usaron las camisas de acero que corresponden a la cuantía mínima (LI 32X3), y se usó otro procedimiento para fijar las camisas, consistente en colocar los ángulos longitudinales, precalentar las soleras y soldarlas a los ángulos mientras estaban calientes. La variable estudiada fue la temperatura a que se precalentaban las soleras. Se usaron cuatro temperaturas: $90^{\circ}, 70^{\circ}, 50^{\circ}$ y temperatura ambiente. Se fabricaron cinco especímenes iguales con cada temperatura por lo que esta segunda serie consistió en 20 ensayes. A continuación se describe el procedimiento constructivo. 
Las soleras se calentaban en un horno eléctrico y se llevaban a una temperatura superior a la especificada, ya que se tomó en cuenta que transcurría aproximadamente un minuto y medio entre el momento de sacarlas del horno y terminar el proceso de soldadura. Así se aseguraba que las soleras estuvieran a la temperatura especificada en el momento de soldarlas. Previamente se colocaban los ángulos en cada una de las aristas y por medio de un anillo de sujeción de acero se fijaban a la columna, figura 5. El procedimiento de precalentar las soleras es quizá el más usado en la práctica, aunque en vez de horno eléctrico se usa gis térmico para controlar la temperatura. Se consideró que en condiciones de laboratorio, el horno eléctrico permitía obtener un mejor control de la temperatura.

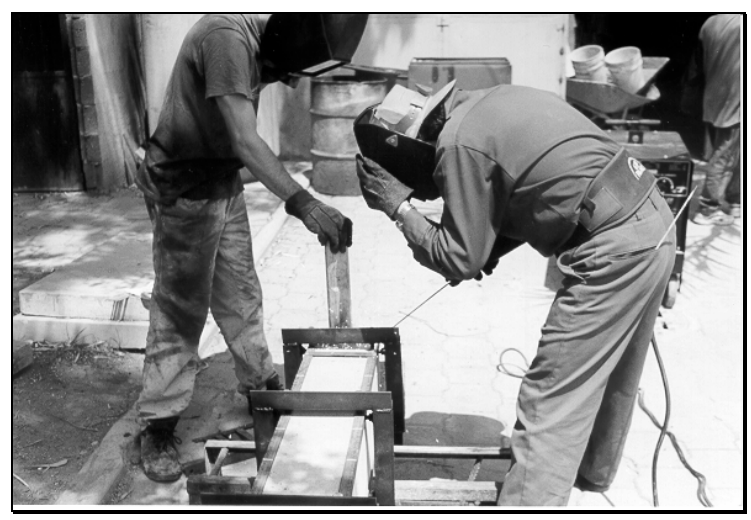

Figura 5. Procedimiento de soldado de las soleras precalentadas

En la tercera serie, se ensayaron especímenes dañados previamente. El daño se ocasionó aplicándoles cargas como las ya descritas, hasta el momento en que se desarrollaban grietas de $0.3 \mathrm{mmm}$ de ancho, que corresponden, como ya se ha señalado, a la carga de agrietamiento visual. Una vez dañados se desmontaban del marco de carga y se les colocaba la camisa de acero, con alguno de los procedimientos descritos para las dos primeras series. En total se elaboraron dieciocho elementos, con las siguientes combinaciones de procedimiento de encamisado.

a) soleras con $90^{\circ}$ de temperatura al momento de soldarlas, con presión y con resina

b) soleras con $90^{\circ}$ de temperatura al momento de soldarlas, con presión y sin resina

c) soleras con $90^{\circ}$ de temperatura al momento de soldarlas, sin presión y con resina

d) soleras con $70^{\circ}$ de temperatura al momento de soldarlas, con presión y con resina

e) soleras con $70^{\circ}$ de temperatura al momento de soldarlas, con presión y sin resina

f) soleras con $70^{\circ}$ de temperatura al momento de soldarlas, sin presión y con resina

g) soleras con $50^{\circ}$ de temperatura al momento de soldarlas, con presión y con resina

h) soleras con $50^{\circ}$ de temperatura al momento de soldarlas, con presión y sin resina

i) soleras con $50^{\circ}$ de temperatura al momento de soldarlas, sin presión y con resina

Se elaboraron dos especímenes con cada una de estas combinaciones. Además, se elaboraron dos elementos testigo sin daño previo y encamisados, lo que hace un total de 20 especímenes. En esta tercera serie sólo se usaron camisas de acero que corresponden a la cuantía mínima. Se descartó también la combinación de variables en la que no se aplican ni presión ni resina, por no considerarla recomendable sobre la base de la primera serie. 


\section{Resultados experimentales}

Serie 1. El comportamiento de la mayoría de los especímenes, con las excepciones mencionadas más adelante, fue similar. Las fallas fueron por tensión diagonal, con deformaciones laterales significativas, o sea, hubo un grado de ductilidad apreciable. Se formaron grietas inclinadas cerca del empotramiento que se fueron prolongando y haciendo más anchas conforme se avanzó en los ciclos de carga. En la figura 6 se muestra una columna típica después de la falla y en la figura 7, su gráfica cargadeformación. El conjunto completo de gráficas se ha presentado en González Cuevas et al. 1999.

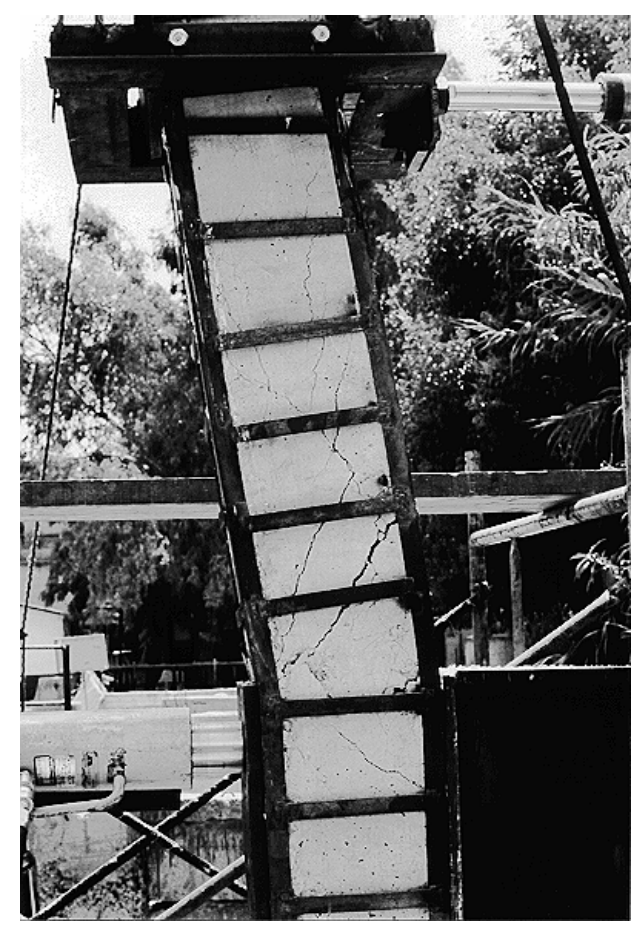

Figura 6. Agrietamiento típico en la falla

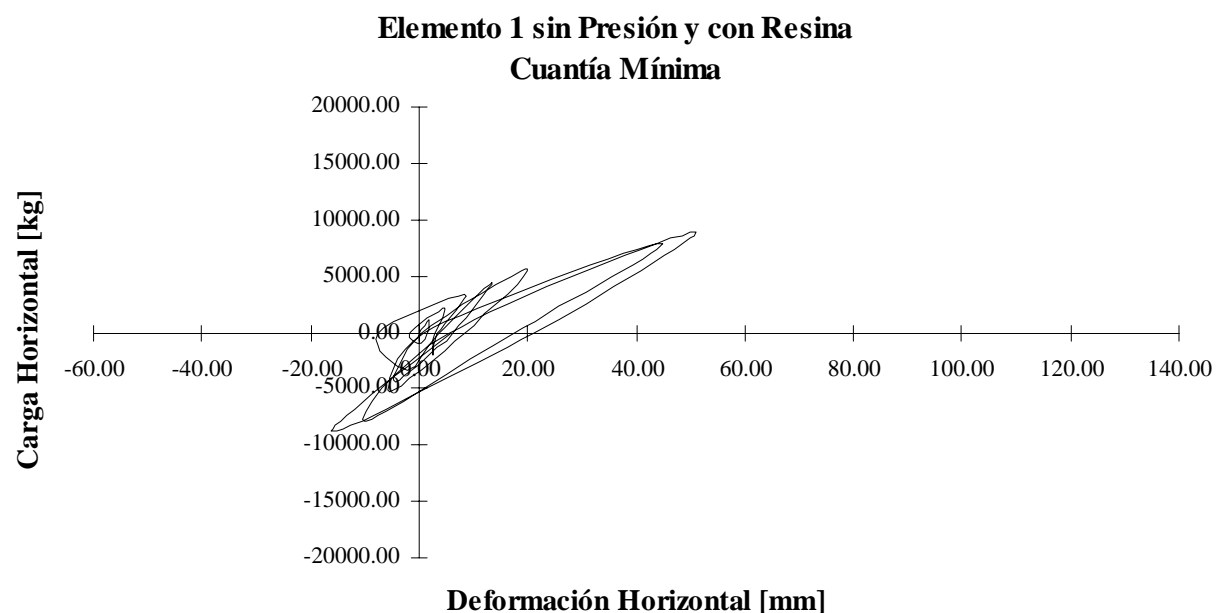

Figura 7. Curva típica esfuerzo deformación lateral 
En ningún caso se observó falla de la soldadura, o de los ángulos o soleras. Falló el concreto en los extremos de la grieta inclinada, en forma similar a las vigas de concreto con refuerzo de estribos que fallan por tensión diagonal. Un comportamiento singular fue el de las columnas sin presión y sin resina. En éstas, se observó un deslizamiento entre la camisa y el concreto de las columnas. En las columnas con cuantía mínima, la resistencia observada fue similar a la de columnas con otros procedimientos de encamisado, pese al deslizamiento. Sin embargo, en las columnas con cuantía máxima, sí hubo reducciones de resistencia en comparación con otros procedimientos o con los elementos testigo, atribuibles al deslizamiento. Este comportamiento se consideró inconveniente, por lo que no se usó el procedimiento correspondiente en las otras series de ensayes. Un fenómeno semejante, pero de menor magnitud, se presentó cuando se usó presión sin resina.

Las cargas de falla registradas se presentan en la tabla 1 (V experimental). La notación empleada es la siguiente. Las tres primeras letras, después de EC, indican si la camisa corresponde a la cuantía mínima o a la máxima (MIN o MAX). Las siguientes letras, si se usó presión para colocar la camisa (P) o resina (R) o ambas (PR); si no hay letra, se trata de un espécimen testigo. El número al final indica si es el primero o el segundo de dos especímenes iguales. Es importante mencionar que en los especímenes ECMINPR-1, ECMINP-1, ECMINP-2, ECMINR-1, ECMINR-2, ECMIN-2, ECMAXP-1, ECMAXP-2, ECMAX-2, ECMAX-1 hubo necesidad de suspender la prueba, por problemas en el equipo de carga, antes de que ocurriese la falla. Por esta razón aparecen con cargas de falla exactamente iguales. Sin embargo, los especímenes con cuantía mínima ya se encontraban muy agrietados por lo que las cargas reportadas deben ser ligeramente inferiores a las que se hubiesen obtenido si se continuaba el ensaye. En los casos de cuantía máxima, el agrietamiento era ligero, con grietas máximas de $0.5 \mathrm{~mm}$, por lo que las cargas experimentales son sustancialmente menores a las de los testigos o a las teóricas.

Tabla 1. Resistencias experimentales y teóricas

\begin{tabular}{ccccc}
\hline Elemento & $\mathbf{f}_{\mathbf{C}} \mathbf{~ k g} / \mathbf{c m}^{\mathbf{2}}$ & $\mathbf{V}$ experimental $\mathbf{( k g )}$ & $\mathbf{V}_{\mathbf{N T C}}(\mathbf{k g})$ & $\mathbf{V}_{\text {TMCC }}(\mathbf{k g})$ \\
\hline ECMINPR-1 & 312 & 8905 & 10467 & 11306 \\
ECMINPR-2 & 312 & 13358 & 10467 & 11306 \\
ECMINP-1 & 218 & 8905 & 9712 & 10819 \\
ECMINP-2 & 218 & 8905 & 9712 & 10819 \\
ECMINR-1 & 218 & 8905 & 9712 & 10819 \\
ECMINR-2 & 248 & 11132 & 9968 & 10984 \\
ECMIN-1 & 231 & 10018 & 9825 & 10892 \\
ECMIN-2 & 240 & 11132 & 9901 & 10941 \\
ECMAXPR-1 & 237 & 15584 & 15739 & 19791 \\
ECMAXPR-2 & 237 & 15584 & 15739 & 19791 \\
ECMAXP-1 & 237 & 11132 & 15739 & 19791 \\
ECMAXP-2 & 298 & 11132 & 16226 & 20027 \\
ECMAXR-1 & 198 & 15584 & 15394 & 19624 \\
ECMAXR-2 & 229 & 13358 & 15670 & 19758 \\
ECMAX-1 & 229 & 8905 & 15670 & 19758 \\
ECMAX-2 & 229 & 11132 & 15670 & 19758 \\
\hline
\end{tabular}

Serie 2. El tipo de falla fue similar al de la serie anterior, es decir, fallas por tensión diagonal con cierta ductilidad. En las columnas en que las soleras se habían calentado a $90^{\circ}$ antes de soldarlas a los ángulos, no se observó ningún deslizamiento entre la camisa de acero y el concreto. Con temperaturas menores, sí hubo deslizamientos, de $1 \mathrm{~mm}$ cuando fue de $70^{\circ}$, de $2 \mathrm{~mm}$ cuando fue de $50^{\circ}$ y de $5 \mathrm{~mm}$ cuando no se calentaron. Sin embargo, los deslizamientos no redujeron las resistencias. En todos los 
especímenes se alcanzó una resistencia de 11,130 kg de fuerza lateral, valor en el cual hubo que suspender los ensayes por problemas de capacidad del equipo de carga. En las figuras 8 y 9 se muestran una falla y una gráfica carga deformación típicas de esta serie. El conjunto completo de gráficas se ha presentado en González Cuevas et al. 2001.

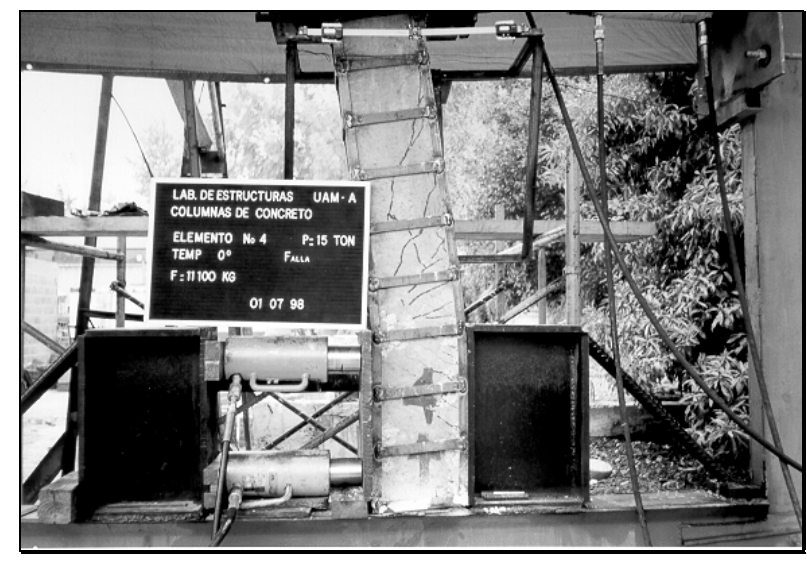

Figura 8. Falla típica de un espécimen de la segunda serie

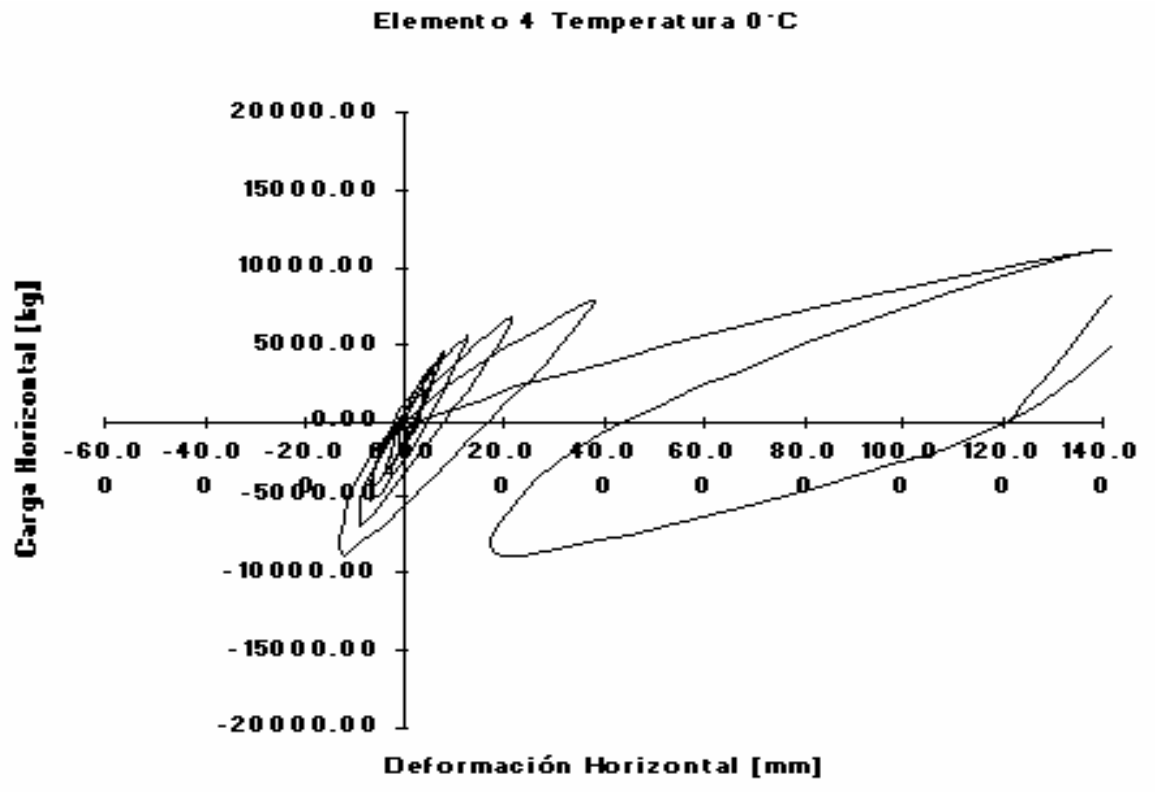

Figura 9. Gráfica fuerza deformación típica de un espécimen de la segunda serie

Serie 3. En esta serie se dañaron previamente los especímenes con grietas de aproximadamente $0.3 \mathrm{~mm}$ de ancho y se encamisaron con soleras calentadas a las tres diferentes temperaturas usadas en la serie anterior y combinando presión y resina. Las fallas fueron también de tensión diagonal, y en todos los casos se sobrepasaron las resistencias teóricas. Sin embargo, en los especimenes en que no se colocó resina se observaron deslizamientos entre las camisas de acero y el concreto, inclusive cuando se calentaron las soleras a $90^{\circ}$. Al igual que en la serie 2, los ensayes se suspendieron a la capacidad máxima del equipo de 11,130 kg de carga lateral. El agrietamiento era severo, con anchos superiores a $3 \mathrm{~mm}$. El 
comportamiento observado fue similar al de columnas sin daño previo. En las figuras 10 y 11 se muestran gráficas con fallas y curvas carga deflexión típicas de esta serie.

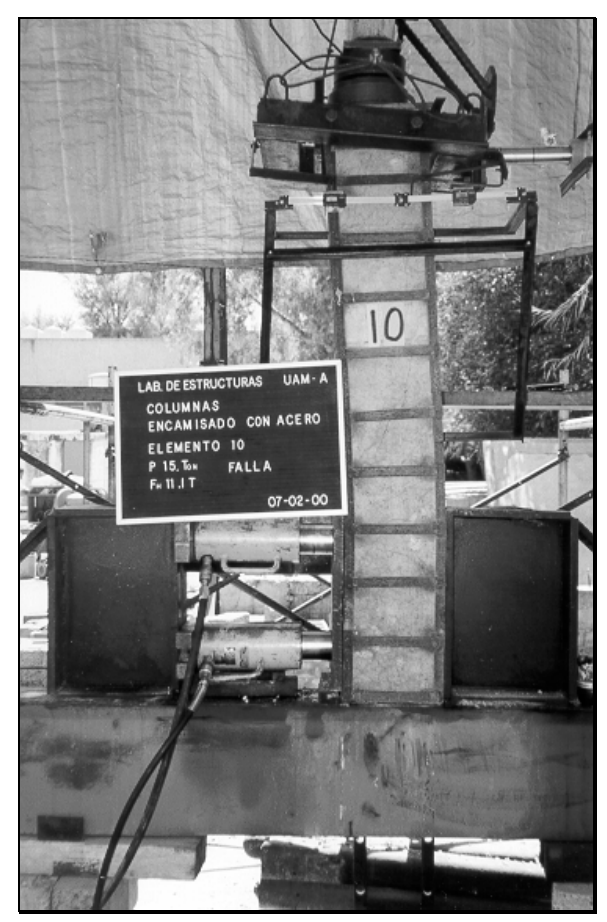

Figura 10. Falla típica de un espécimen de la tercera serie

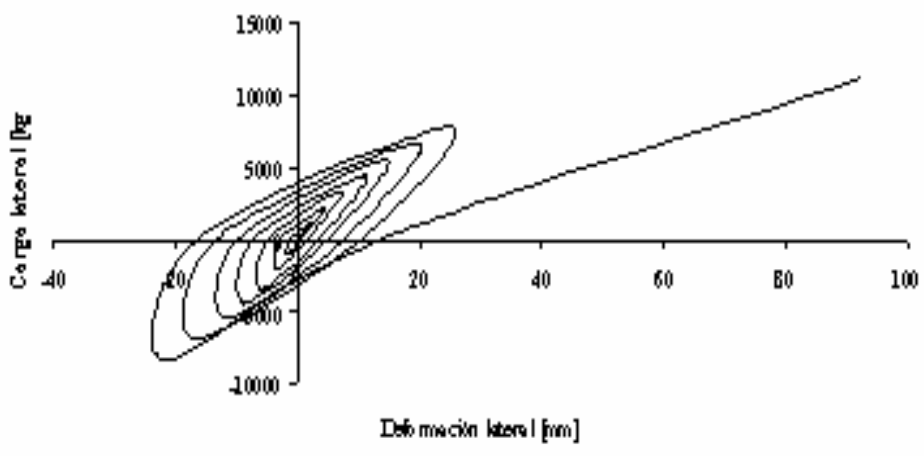

Figura 11. Gráfica fuerza deformación típica de un espécimen de la tercera serie

\section{ANÁLISIS DE RESULTADOS}

Los resultados se analizaron desde el punto de vista de la resistencia y de la ductilidad de los elementos, aunque ya que las fallas fueron de tensión diagonal, no se esperaba una ductilidad grande. Para hacer una comparación entre las resistencias teóricas de las columnas y las obtenidas experimentalmente, se calcularon sus resistencias a fuerza cortante y sus diagramas momento - curvatura. Al obtener los 
diagramas momento - curvatura fue posible establecer la máxima carga que podría ser aplicada en el elemento de concreto para asegurar que su falla fuera por cortante y no por momento. Asimismo fue posible calcular la deformación teórica que experimentaría cada elemento. A continuación, se presentan los resultados obtenidos.

\section{Resistencia a fuerza cortante}

Para el cálculo de la resistencia a cortante de cada tipo de columnas se utilizaron las expresiones establecidas en las NTCC - 04, tomando en cuenta el efecto de la carga axial, sin aplicar el factor de reducción de resistencia $F_{R}$, y usando la resistencia del concreto $f_{c}^{\prime}$ en vez de la resistencia reducida $f_{c}^{*}$. Se consideró que las soleras trabajaban igual que un estribo interior, por lo que su contribución a la resistencia a fuerza cortante se calculó con la ecuación usual de la analogía de la armadura. A continuación se muestran los resultados.

Datos

$\mathrm{b}=20 \mathrm{~cm}$

$\mathrm{h}=25 \mathrm{~cm}$

$\mathrm{d}=21.55 \mathrm{~cm}$

$\mathrm{P}_{\mathrm{u}}=15$ ton

$f_{c}^{\prime}$ variable; los valores se muestran en la tabla 1.

Las ecuaciones a utilizar son la 1 a la 3 :

$\mathrm{V}_{\mathrm{cr}}=0.5 \mathrm{~b} \mathrm{~d}\left(\mathrm{f}_{\mathrm{c}}\right)^{1 / 2}\left[1+0.007\left(\mathrm{P}_{\mathrm{u}} / \mathrm{A}_{\mathrm{g}}\right)\right]$

$V_{s}=A_{v} f_{y} d / s$

$\mathrm{V}_{\text {tot }}=\mathrm{V}_{\mathrm{cr}}+\mathrm{V}_{\mathrm{s}}$

Los valores teóricos obtenidos de esta manera se muestran en la tabla 1 en la columna $V_{\mathrm{NTC}}$.

Las tres columnas testigo que se probaron, una por serie, tenían únicamente un estribo a la mitad de la altura y otros dos en los extremos, que se colocaron para fines de fabricación de los especímenes. Por lo tanto, teóricamente no existía contribución de los estribos a la resistencia total. Sin embargo, la resistencia experimental de estas columnas fue en promedio de $6200 \mathrm{~kg}$, mucho mayor que la resistencia calculada $\mathrm{V}_{\mathrm{cr}}$. La única explicación que se encontró para justificar esta diferencia es que en todos los casos se formó una grieta que atravesaba el único estribo. Este estribo podría entonces haber contribuido a la resistencia total.

Ya se ha comentado que en algunos especímenes con la cuantía mínima fue necesario suspender la prueba cuando la carga lateral, que es igual a la fuerza cortante, había alcanzado un valor de 8,905 kg, o sea, del 85 al 92 por ciento de la resistencia teórica y que el nivel de daño era todavía pequeño. En el resto de los especímenes con cuantía mínima, la resistencia alcanzada, todavía menor que la de falla, fue de 11,132 kg, o sea, 12 por ciento mayor que las teóricas. Esto es válido para las tres series de ensayes, es decir, aun los especímenes con daño previo alcanzaron resistencias a fuerza cortante superiores a las teóricas, o casi iguales cuando se suspendió el ensaye antes de la falla.

En los especímenes con cuantía máxima, se alcanzaron resistencias hasta de 15,548 kg, valor prácticamente igual a las resistencias teóricas, sin producirse la falla y con daños ligeros. 
A partir de esta comparación entre resistencias teóricas y experimentales se puede concluir que las columnas encamisadas con cualquiera de los procedimientos utilizados en esta investigación tienen resistencias mayores que las calculadas con las ecuaciones de las NTCC del Reglamento del Distrito Federal. Inclusive las columnas con daño previo consistente en grietas inclinadas de hasta $0.3 \mathrm{~mm}$ de ancho sobrepasaron las resistencias teóricas.

Sin embargo, debe tenerse presente que con algunos procedimientos se observaron deslizamientos entre la camisa de acero y el concreto, lo cual es indeseable desde el punto de vista del comportamiento estructural. En general, el uso de resina epóxica para pegar las camisas al concreto evita este deslizamiento.

Con el fin de tener otro valor teórico de la resistencia a fuerza cortante, se consideró conveniente calcular dicha resistencia con un método alternativo al de las NTCC. Para esto se utilizó el Método de la Teoría Modificada del Campo de Compresión (Vecchio y Collins, 1986), el cual toma en cuenta otras variables importantes, como la inclinación de las grietas diagonales, $\theta$, y las deformaciones longitudinales del elemento al nivel del acero de tensión. Este método se considera como un avance significativo en el estudio de la tensión diagonal (ACI-ASCE Committee 445, 1999).

Según este método, la resistencia a fuerza cortante puede calcularse con la ecuación 4:

$$
V_{n}=\beta \sqrt{f_{c}^{\prime}} b_{w} d+\frac{A_{s} f_{y}}{s} d \cot \theta
$$

El parámetro $\beta$ y la inclinación de las grietas, $\theta$, dependen, a su vez, de la deformación unitaria al nivel del acero de tensión y de la relación $v_{u} / f_{c}^{\prime}$ entre el esfuerzo cortante promedio y la resistencia a compresión del concreto. Las resistencias calculadas de esta manera se indican también en la tabla 1 en la

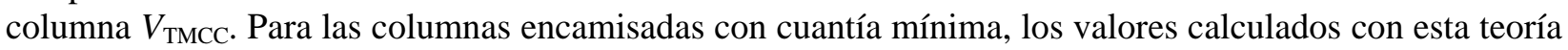
resultaron semejantes a los calculados con las NTCC, y para las columnas con cuantía máxima, aproximadamente 30 por ciento mayores. Tomando en cuenta que el nivel de daño era ligero cuando se suspendieron las pruebas, se estima que las columnas hubiesen podido alcanzar las fuerzas cortantes calculadas con esta teoría.

Es interesante señalar que el ángulo de inclinación de las grietas calculado con la Teoría Modificada del Campo de Compresión resultó de $39^{\circ}$ con cuantía mínima y de $40^{\circ}$ con cuantía máxima. Estos valores son significativamente diferentes al de $45^{\circ}$ supuesto en la analogía clásica de la armadura de Ritter. Si se observan las fotografías de las figuras 6, 8 y 10 se puede ver que la inclinación de las grietas de los especímenes fallados es más parecida a las predichas con la Teoría Modificada del Campo de Compresión que a $45^{\circ}$. En esta teoría se atribuye a la inclinación de las grietas un papel importante para explicar la diferencia entre las resistencias calculadas con la teoría clásica y las experimentales.

\section{Diagramas Momento - Curvatura}

Se obtuvieron diagramas momento-curvatura de las secciones de los especímenes utilizando un programa de cómputo elaborado por los autores. En dicho programa se considera a la zona de compresión del concreto discretizada en pequeñas porciones. En cada una de ellas se calculan las deformaciones unitarias y con el Modelo de Kent y Park Modificado (Kent, 1971; Scott, Park y Priesley, 1982), se obtienen los esfuerzos a los que se encuentran sometidas. Cabe aclarar que este modelo considera el efecto del confinamiento proporcionado al concreto por los estribos o por las soleras. Para cada tipo de 
espécimen se calcularon dos diagramas distintos que difieren en el modelo utilizado para describir el comportamiento del acero. En uno de ellos, denominado teórico, se considera que el acero fluye indefinidamente al alcanzar una deformación unitaria de 0.002, mientras que en el segundo, denominado real, se considera que el acero tiene la gráfica esfuerzo deformación propuesta por Rodríguez y Botero (1995), la cual incluye una zona de endurecimiento por deformación. En ambos casos se considera que el elemento falla cuando se aplasta el concreto. Por lo que respecta a la contribución de los ángulos en las columnas encamisadas, se consideró un área equivalente debido a la diferencia entre los esfuerzos de fluencia del acero de refuerzo y del acero estructural.

Los diagramas momento - curvatura obtenidos de la manera descrita se presentan en las figuras 12 y 13 para algunos elementos con cuantías mínima y máxima, respectivamente.

\section{Diagrama Momento - curvatura \\ Elemento con Cuantía mínima \\ f 'c experimental}

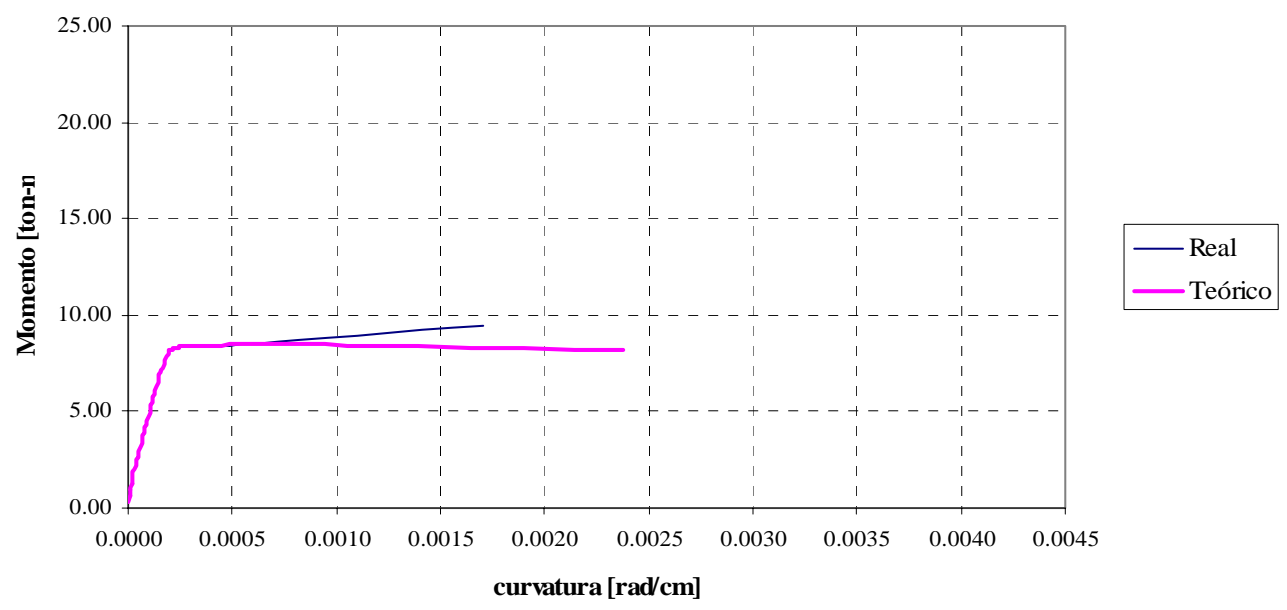

Figura 12. Diagramas momento-curvatura para cuantía mínima

A partir de estos diagramas momento-curvatura se calcularon las gráficas carga-deflexión teóricas de los especímenes, hasta alcanzar las cargas correspondientes a las fallas teóricas por fuerza cortante. Las deflexiones se obtuvieron integrando las curvaturas correspondientes a los momentos flexionantes a lo largo de las columnas, sin considerar zonas de articulación plástica, ya que las curvaturas correspondientes a las cargas de falla por cortante eran pequeñas. En las figuras 14 y 15 se presentan estas gráficas. Puede verse que las deflexiones teóricas calculadas de la manera descrita son mucho menores que las experimentales, figuras 9 y 11. Esto se debe a que las cargas de falla fueron también mayores que las teóricas por lo que en los diagramas momento-curvatura se había ingresado a las zonas inelásticas.

\section{Disipación de energía y degradación de rigidez}

Ya se ha señalado que debido al tipo de falla, no se esperaba que estos especímenes de ensaye exhibiesen una rama amplia de comportamiento inelástico. De todas maneras, se analizó este comportamiento a través de dos parámetros: la disipación de energía y la degradación de rigidez. 
Para analizar el primero, se obtuvieron las áreas dentro de cada ciclo histerético de las gráficas cargadeflexión, que representan la energía disipada en cada uno. Esto se hizo con un método numérico a partir de las deflexiones medidas. Con las áreas calculadas se trazaron gráficas del área contra el número de ciclos. En las figuras 16 y 17 se muestran la gráficas que corresponden a los especímenes MINPR-2 y MINP-1 de la tabla 1. Comparando estas gráficas se puede ver que la disipación de energía es muy semejante en ambas, lo que indica que el procedimiento de fabricación de la camisa no influyó en este parámetro. Otras gráficas pueden verse en (Muñoz, 2000).

\section{Diagrama Momento - curvatura \\ Elemento con Cuantía máxima f 'c expenimental}

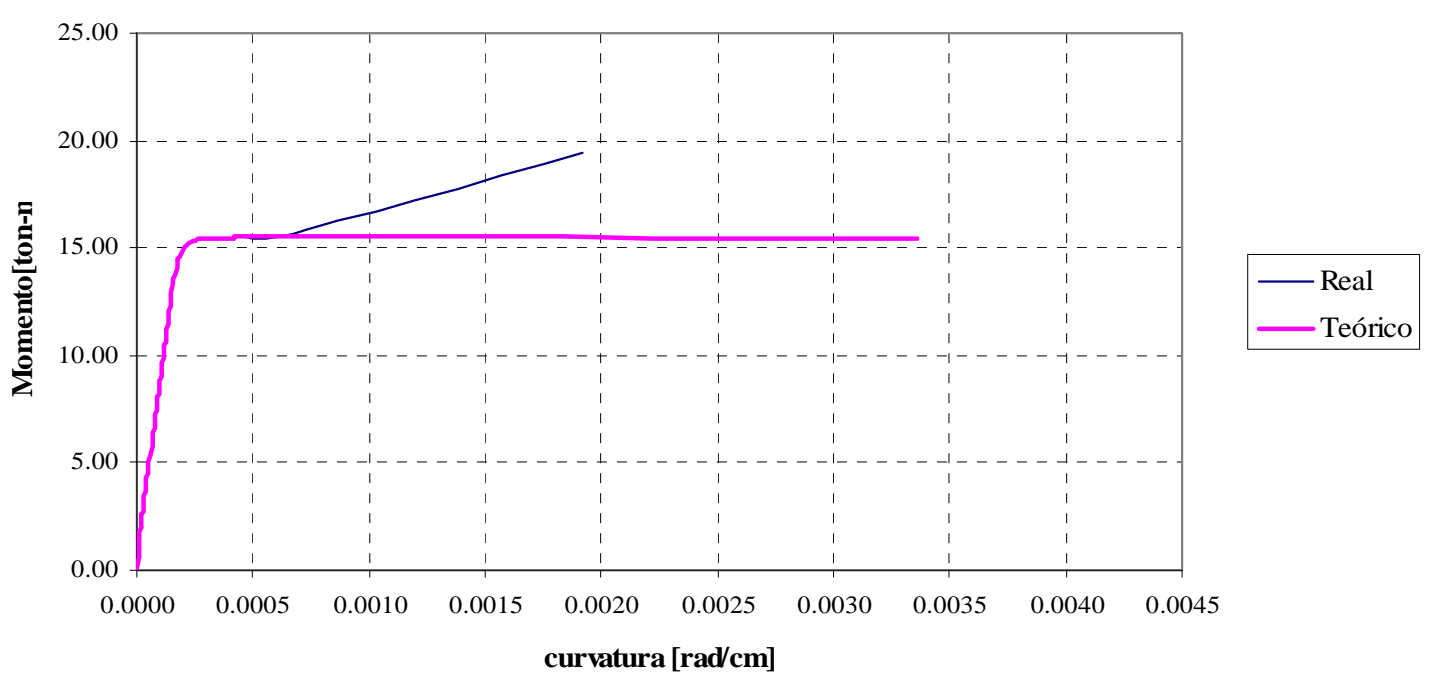

Figura 13. Diagramas momento-curvatura para cuantía máxima

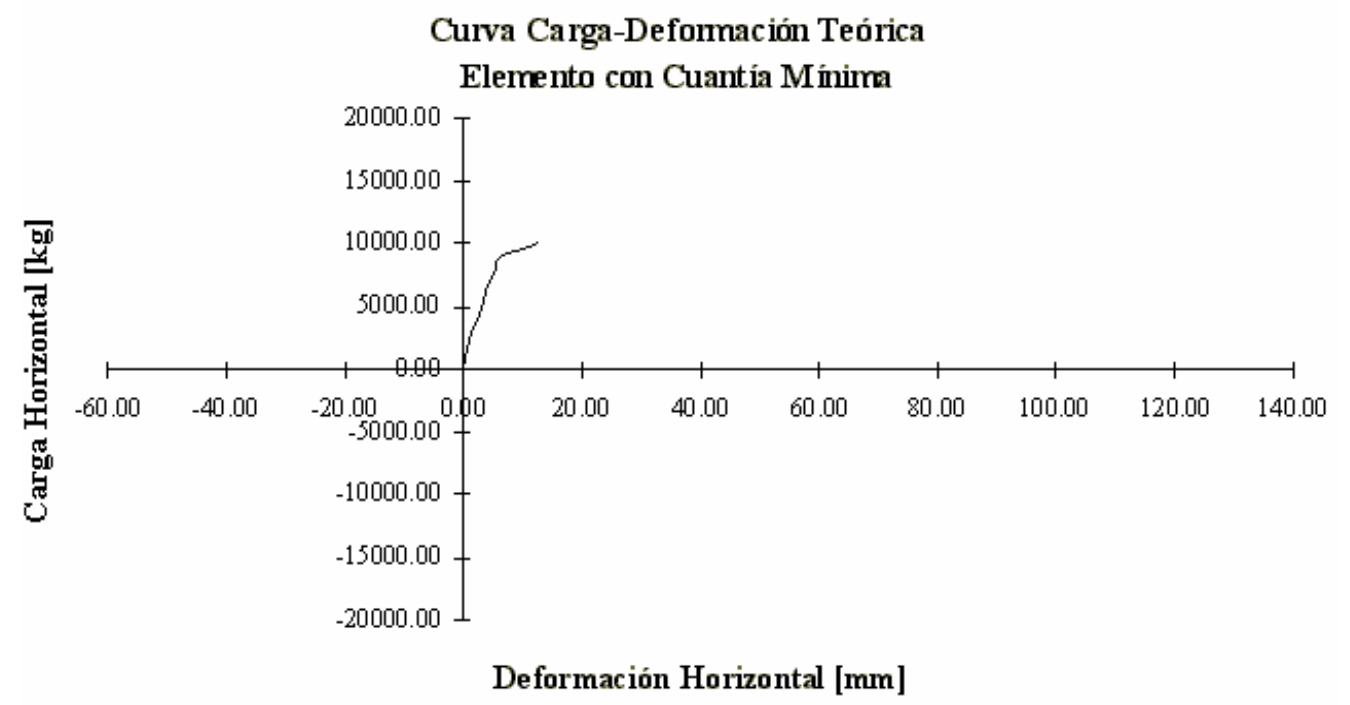

Figura 14. Gráfica carga-deflexión teórica para cuantía mínima 


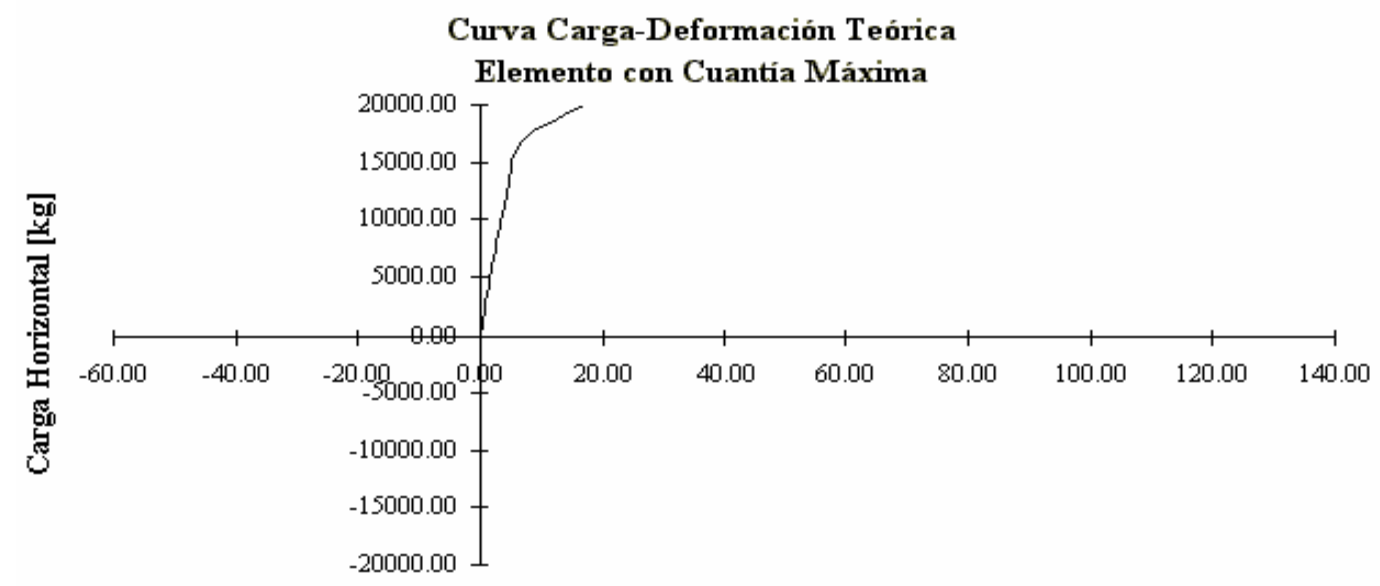

Deformación Horizontal [mm]

Figura 15. Gráfica carga-deflexión teórica para cuantía máxima

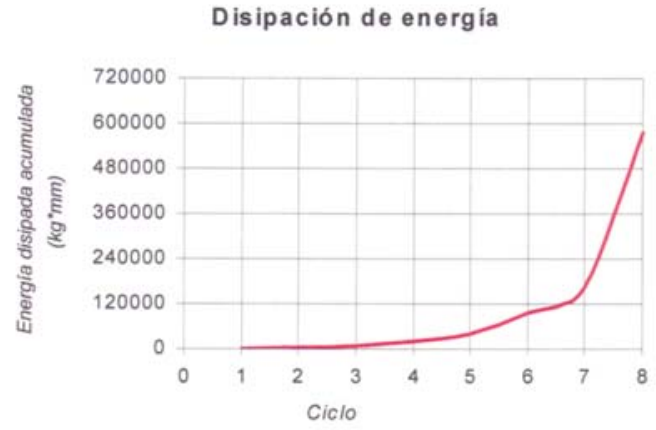

Figura 16. Espécimen MINPR-2

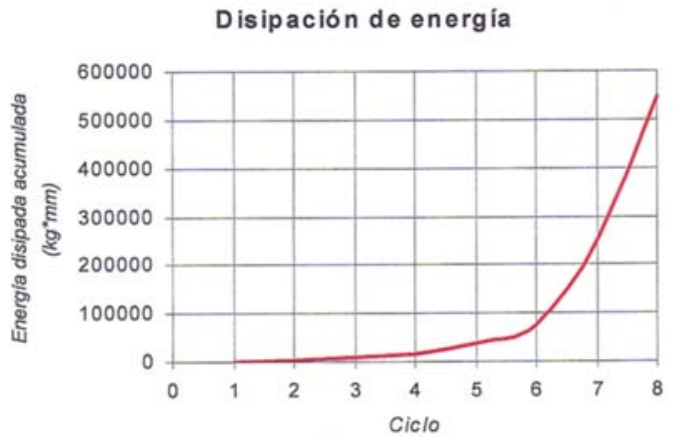

Figura 17. Espécimen MINP-1

La degradación de rigidez se obtuvo calculando la rigidez de cada ciclo como la pendiente de la recta que une el origen de la gráfica con la carga máxima del ciclo, y trazando la gráfica de rigidez de ciclo contra distorsión; esta última se define de la manera usual como el cociente entre la deflexión lateral y la altura de la columna. En las figuras 18 y 19 se muestran las gráficas correspondientes a los dos especímenes mencionados en el párrafo anterior. En este caso sí se observa que la degradación de rigidez fue mayor cuando no se usó resina.

\section{CONCLUSIONES}

Los distintos procedimientos estudiados en esta investigación, para encamisar columnas de concreto con ángulos longitudinales y soleras transversales de acero, resultaron satisfactorios. Las resistencias a fuerza cortante de los especímenes ensayados con cargas cíclicas resultaron iguales o mayores que las calculadas con las ecuaciones de las NTCC del Reglamento del Distrito Federal, considerando que las 
soleras transversales trabajan como si fuesen estribos interiores. Sin embargo, cuando no se usó resina epóxica para pegar los ángulos al concreto, se observaron deslizamientos entre las camisas y el concreto. Este deslizamiento no redujo la resistencia, pero sí se observó una mayor degradación de rigidez ante ciclos repetidos de carga en los especímenes sin resina. Se recomienda, por lo tanto, pegar los ángulos al concreto con resina epóxica siempre que sea posible.

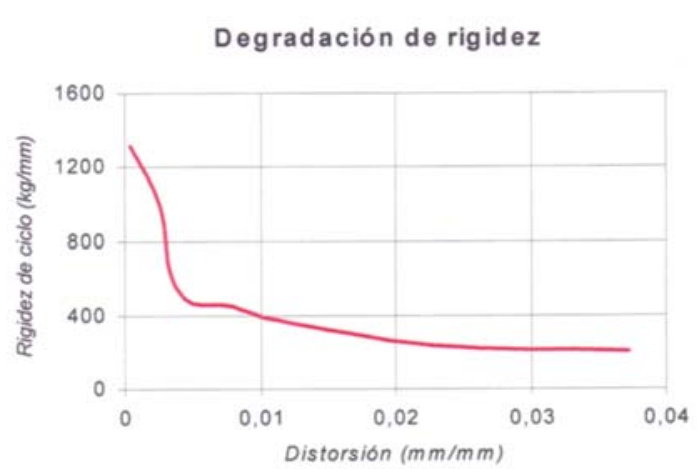

Figura 18. Espécimen MINPR-2

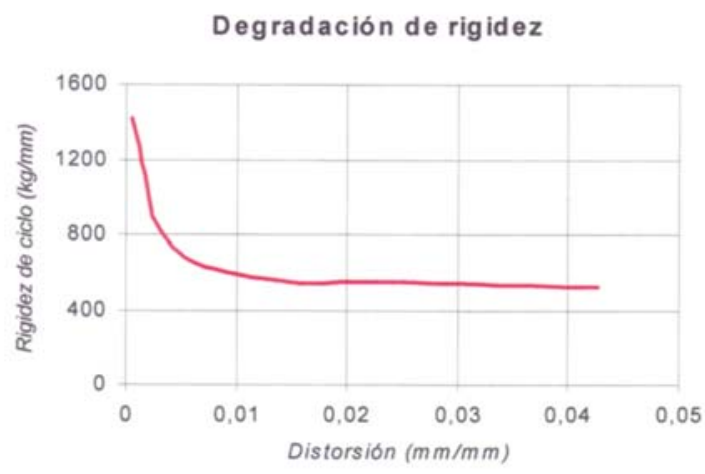

Figura 19. Espécimen MINP-1

Las columnas encamisadas después de haber sufrido daños previos también alcanzaron resistencias a fuerza cortante mayores que las teóricas. Los daños previos consistieron en grietas inclinadas de aproximadamente $0.3 \mathrm{~mm}$ de ancho, que corresponden a la carga visual de agrietamiento inclinado. Se puede recomendar, por lo tanto, que las columnas que hayan sido dañadas por sismo, con daños similares a los estudiados, se refuercen con camisas de acero para incrementar su resistencia a fuerza cortante.

\section{REFERENCIAS}

ACI-ASCE Committee 445 (1999), Recent approaches to shear design of structural concrete. American Concrete Institute, Detroit.

Gobierno del Distrito Federal (2004), Normas técnicas complementarias para diseño y construcción de estructuras de concreto. Gaceta Oficial del Distrito Federal, Tomo I, No. 103 bis, 6 de octubre de 2004.

González Cuevas, O, J J Guerrero Correa, B Gómez González y F Flores Díaz (1999), "Resistencia a fuerza cortante de columnas de concreto reforzado con camisas de acero - $1^{\text {a }}$. etapa”. Construcción y Tecnología, IMCYC. Vol. 11, No. 129, febrero 1999.

González Cuevas, O, J J Guerrero Correa, B Gómez González, F Flores Díaz (2001), “Resistencia a fuerza cortante de columnas de concreto reforzado con camisas de acero $-2^{\mathrm{a}}$. etapa”, Construcción y Tecnología. IMCYC. Vol. 14. No. 152, enero 2001.

González Cuevas, O y F Robles (2005), Aspectos fundamentales del concreto reforzado, Cuarta edición, LIMUSA, México, 802 pp.

Iglesias, J, F Robles, J de la Cera y O González Cuevas (1988), Reparación de estructuras de concreto y mampostería, Universidad Autónoma Metropolitana, Unidad Azcapotzalco, México, 145 pp. 
Kent, D C y R Park (1971), "Flexural members with confined concrete”, Proceedings ASCE, Vol. 97, ST 7, July, pp. 1969-1990.

Muñoz, B (2000), “Disipación de energía y degradación de rigidez en columnas encamisadas”, Proyecto terminal de Ingeniería Civil, UAM-Azcapotzalco, México.

Rodríguez, M, y J C Botero (1995), “Comportamiento sísmico de estructuras considerando las propiedades mecánicas de acero de refuerzo fabricado en México”, Revista de Ingeniería Sísmica, No. 49, pp 39-50.

Scott, B D, R Park y M J N Priestley (1982), “ Stress-strain behavior of concrete confined by overlapping hoops at low and high strain rates”, ACI Structural Journal, January-February, pp. 13-27. American Concrete Institute, Detroit.

Vecchio, F J y M P Collins (1986), “The modified compression field theory for reinforced concrete elements subjected to shear “, ACI Journal, Proceedings Vol. 83, No. 2, Mar- Apr., pp 219-231. 